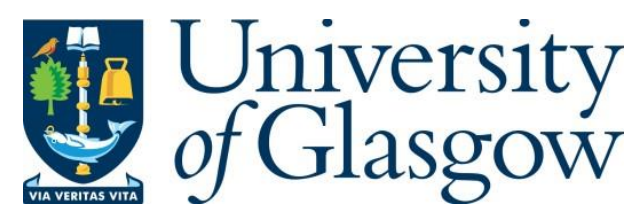

Ng, A. and Brewster, S. (2017) An Evaluation of Touch and Pressure-Based Scrolling and Haptic Feedback for In-car Touchscreens. In: AutomotiveUI 2017, Oldenburg, Germany, 24-27 Sep 2017, pp. 11-20. ISBN 9781450351508.

There may be differences between this version and the published version. You are advised to consult the publisher's version if you wish to cite from it.

http://eprints.gla.ac.uk/144669/

Deposited on: 25 July 2017

Enlighten - Research publications by members of the University of Glasgow http://eprints.gla.ac.uk 


\title{
An Evaluation of Touch and Pressure-Based Scrolling and Haptic Feedback for In-car Touchscreens
}

\author{
Alexander Ng and Stephen Brewster \\ Glasgow Interactive Systems Section \\ School of Computing Science \\ University of Glasgow, Glasgow, UK \\ \{first.last\}@glasgow.ac.uk
}

\begin{abstract}
An in-car study was conducted to examine different input techniques for list-based scrolling tasks and the effectiveness of haptic feedback for in-car touchscreens. The use of physical switchgear on centre consoles is decreasing which allows designers to develop new ways to interact with incar applications. However, these new methods need to be evaluated to ensure they are usable. Therefore, three input techniques were tested: direct scrolling, pressure-based scrolling and scrolling using onscreen buttons on a touchscreen. The results showed that direct scrolling was less accurate than using onscreen buttons and pressure input, but took almost half the time when compared to the onscreen buttons and was almost three times quicker than pressure input. Vibrotactile feedback did not improve input performance but was preferred by the users. Understanding the speed vs. accuracy trade-off between these input techniques will allow better decisions when designing safer incar interfaces for scrolling applications.
\end{abstract}

\section{Author Keywords}

In-car interaction; Input techniques; Haptic feedback; Pressure input; Touch Input; Touchscreens.

ACM Classification Keywords

H.5.2 User Interfaces (Input devices and strategies)

\section{INTRODUCTION}

Modern cars allow drivers to use in-car controls and interact with systems in many new ways with the use of touchscreens and gesture detection hardware on centre consoles (e.g. BMW Touch and Gesture Command [4]). This gives designers the opportunity to develop new input techniques and applications that could be more effective and safer to use over standard physical switchgear while driving. Previous work has studied different types of input with in-car touchscreens (e.g. $[2,6])$ and more recently pressure- based interactions on in-car touch-sensitive surfaces (e.g. $[13,20,21])$ as force input is becoming more common with popular touchscreen mobile devices such as the Apple iPhone. Pressure-based input is likely to feature in cars in the future and we test its performance with touchscreens for list scrolling applications, something that is less well studied.

This paper presents an in-car non-driving study which compares touch- and pressure- based input techniques for common scrolling tasks on in-car touchscreens. Many tasks such finding a song in a playlist or searching for a location in the GPS application may need significant scrolling actions and can take a considerable amount of time to complete. As a result, drivers are likely to be distracted from driving in a safe manner when interaction is required. A more efficient input method for scrolling lists could reduce input time and thus attention taken away from looking ahead on the roads. Therefore, we tested three types of controls for common scrolling tasks on a touchscreen: (1) direct scrolling (2) rate-based pressure scrolling and (3) scrolling using onscreen buttons. Users sat inside a moving vehicle to evaluated the input techniques so that they experience the forces, vibrations and movements of typical driving situations.

One limitation with replacing physical buttons and dials with touchscreens is the lack of haptic sensations. Most current in-car touchscreens lack any form of haptic feedback to inform users a touch event or action has happened. Therefore we also evaluated the different types of input techniques with and without simple vibrotactile cues to investigate if such feedback had any effects on input performance while on the move.

\section{BACKGROUND}

A body of research has studied different types of touch input on centre consoles as touchscreens have become more widespread in cars $[6,8,14,16,26]$. Previous work has examined the effects of scrolling tasks on driving performance. Lee et al. [17] conducted a driving simulator study which varied list lengths for scrolling with an iPod device (4-inch touchscreen) and found that driving performance decreased in terms of lane deviation. Increasing list length also caused longer glance time (greater than 2 s was reported), hence less visual attention was used on the roads ahead. A third-party dial was also evaluated to scroll the interface indirectly and results showed that it caused more glances 
away from the road than scrolling directly on the iPod's touchscreen.

Kujala [15] compared three different scrolling techniques (onscreen buttons, swipe and kinetic) on a touchscreen device and evaluated the effects input had on driver distraction. The results from carrying out a driving simulator study showed that kinetic scrolling required more visual attention and an increase in workload when compared to page-by-page swiping and using onscreen buttons.

Later, Lasch and Kujala [16] evaluated the same three scrolling techniques but also varied the number of items shown onscreen per page and using a physical dial for indirect input. The results from their simulated driving study found that swiping caused fewer number of glances at the 4-inch touchscreen and shorter duration per glance than using the onscreen buttons and the kinetic technique. Error rate (selecting the wrong item in the list) was high across all three techniques, up to $35 \%$ was reported for kinetic scrolling. But the key finding from their study was that scrolling tasks and applications cause great visual demands on the driver as "Total in-vehicle glance times exceeded current NHTSA recommendation [1] of 12 seconds by two to five times". Better methods to interact with in-car list interfaces is likely to reduce driver distraction and on-road safety.

Pressure-based input with touchscreen mobile phones and tablets in non-driving contexts has been well researched (e.g. [5, 9, 10, 18, 19, 24, 29]). Ramos et al. [24] reported that up to six pressure levels could be controlled before performance decreased when targeting on a graphics tablet using a stylus. McLachlan et al. [18] studied bimanual interactions with touchscreen tablets and reported that accuracy for pressure input was high when using the nondominant hand and found a low effect on the dexterity of the dominant hand. Later, McLachlan and Brewster [19] investigated the effects of pressure input using the nondominant hand on a set of common multi-touch gestures (such as taps, swipes, pinches etc.) performed using the dominant hand. The results showed that users could keep high pressure input accuracy without causing increased error in pointing precision. Research has also looked at the use of pressure-based input with mobile phones for side-ofdevice interactions (e.g. Hoggan et al. [11] and Spelmezan et al. [28]). These results were obtained for handheld devices but pressure input is less well studied with in-car touchscreen scrolling interfaces where extra vibrations, movements and forces from a moving vehicle may affect input and driving performance in different ways to nondriving contexts.

More recently, Huber et al. [8] carried out an elicitation study which looked at different force-based gestures on a touchpad for in-car commands. From their driving simulator study, they found that users tend to use two levels of force to differentiate between a tap and a press to interact with in-car applications.
In our previous work [20], we examined the performance of different pressure-based input techniques on a touch-surface and compared the results with a physical dial for abstract menu targeting tasks. In our first experiment using a driving simulator, two common pressure input methods, Positional and Rate-based controls, were evaluated and results showed that positional pressure input caused more incorrect selections and took longer than both rate-based control and the physical dial. Lane deviation was also found to be greater during positional pressure input than the other two input methods. In our second experiment where users sat inside a moving vehicle to examine how the input techniques performed in real world in-car situations, accuracy was high for all input methods $(>98 \%)$ but selection times for all input methods took longer than when compared to the results from the driving simulator experiment.

Later, we investigated four touch- and pressure- based input techniques for a non-scrolling, list-based targeting task while users drove on public roads [21]. The results showed that accuracy was greater than $94 \%$ across all input techniques. Pressure-based buttons on a touch-surface was the slowest method while direct selection on the touchscreen was quicker than using onscreen arrow buttons and a physical dial. Pressure input also caused more glances at the touchscreen than the other three input techniques but the duration per glance was shorter whereas direct selection resulted in the least number of glances but each glance was slightly longer. This trade-off shows that interaction with touchscreens and controls will cause some form of driver distraction. It is important to ensure that new techniques and applications miminise the amount of attention taken away from the roads and drivers control the vehicle in a safe manner.

Research that has examined the effectiveness of haptic feedback for in-car touchscreens such as Pitts et al. [17] found that combining visual, auditory and haptic modalities was subjectively preferred over visual feedback only. Richter et al. [19] conducted a simulated driving study and found that haptic feedback helped reduce error rate and improve task completion time on a force-sensitive touchscreen device. In our previous studies [20,21] where we looked at touch- and pressure- based input techniques, vibrotactile feedback improved both accuracy and selection time when selecting targets in a list in simulated driving and in-car situations. Our work also found that haptic feedback reduced visual distraction from looking on the roads and was preferred by users [21]. Therefore, haptic feedback was tested in the experiment reported in this paper to see if it could improve scrolling performance while inside a moving vehicle.

\section{IN-CAR SCROLLING STUDY}

We conducted an in-car study to compare the performance of three main input techniques on a centre console touchscreen and examined the effects of varying target size on a list-based scrolling task. The three input techniques 
were: (1) directly scrolling and selecting targets in a list (Direct Touch), (2) using onscreen buttons (Onscreen Buttons) and (3) using rate-based pressure buttons (Pressure Input). The input techniques were also tested with and without vibrotactile feedback to see if it improved targeting performance while users sat inside a moving vehicle.

\section{Scrolling Task}

A list-based scrolling task was designed on a touchscreen tablet to replicate common list navigation tasks such as browsing a music collection. We selected a total of 1000 songs from a Spotify playlist and then randomly chose 50 songs for each experimental condition to reduce learning effects. The songs were displayed alphabetically from the top of the touchscreen to the bottom. For each condition, participants selected 15 songs from the list so that the experiment would finish within the set time limit of 90 minutes. The songs to select for each condition were pseudo-randomly chosen as follows. The ordered list of 50 songs was divided into five groups of ten and then three songs were randomly chosen from each group. This meant that participants selected a range of tracks throughout the list for each condition and allowed a fairer comparison between the input techniques.

Two menu item heights were tested: $4 \mathrm{~mm}$ and $8 \mathrm{~mm}$. The height of $8 \mathrm{~mm}$ was based on the dimensions of items in the list interface from a BMW (2015 2 Series Coupe) and a VW (2011 Scirocco) touchscreen system. We also wanted to test the effects of reducing the menu item height, thus showing more items on the screen, on targeting performance for each input technique, so decided to evaluate menu item heights half the size of $8 \mathrm{~mm}$. The total area used for the list of songs was $165 \times 120 \mathrm{~mm}$, which meant a maximum of 15 and 30 items could be shown onscreen for item heights of $8 \mathrm{~mm}$ and $4 \mathrm{~mm}$ respectively. This also allowed space to display the current song to select (above the list) and the onscreen touch- and pressure- based buttons (placed below the list). See Figure 1 (left) for a screenshot of the scrolling task for the input techniques.

A 750ms beep indicated the start of each trial and participants were asked to select the song displayed at the top of the screen as quickly and as accurately as possible. A random gap of between 5 - 10s was placed between each trial to break up any input rhythm. The task ran on a Microsoft Surface Pro 3, which has a touchscreen dimension of 12 inches and $1440 \times 2160 \mathrm{px}(8.5 \mathrm{px} / \mathrm{mm})$ when positioned in portrait mode. Cars such as the Tesla Model X have a similar touchscreen form factor.

For Direct Touch, participants scrolled the list and selected the song directly on the touchscreen. For Onscreen Buttons, bi-directional arrow buttons were placed below the list of songs for scrolling. There were 'UP' and 'DOWN' buttons to move a cursor through the list and an ' $\mathrm{OK}$ ' button to select the target (see Figure 1). Each button measured $30 x$ $30 \mathrm{~mm}$, similar to our previous studies [20]. The cursor was always placed at the top of the list for each trial and tapping on the list directly was disabled to avoid accidental selections when the onscreen buttons were used. Participants had to lift off the arrow buttons each time for the cursor to move by one position, just like a physical button.

For Pressure Input, two $16 \mathrm{~mm}$ Peratech force sensor (www.peratech.com) were mounted on the back of the tablet. Participants pressed on the same arrow buttons as $\mathrm{On}$ screen Buttons, but a minimum pressure of $3 \mathrm{~N}$ had to be detected before the cursor moved. The threshold was chosen after initial pilot tests and followed our previous pressure input studies [20, 21]. Pressure Input was rate-based, so a pressure range from 3 - $6 \mathrm{~N}$ was directly mapped to the speed of the cursor in terms of refresh rate (a range of $2-6$ frames per second). We decided to reduce the pressure input range and overall speed of the cursor from our previous work [20] to decrease overshooting targets and reduce physical fatigue. To select an item in the list, participants simply pressed the 'OK' button as in the Onscreen Buttons condition, no pressure detection was required. There was no looping back if the cursor reached either end of the list for Onscreen Buttons and Pressure Input.

Related work (e.g. [7, 12, 22, 23, 25, 27]) has found that touch input with in-car interfaces improved with the addition of haptic feedback, so we tested the input techniques with vibrotactile cues to see if it influenced targeting performance. One Adafruit large surface transducer (https://www.adafruit.com/product/1784) was securely attached to the touchscreen and a $200 \mathrm{~ms}, 200 \mathrm{hz}$ sine wave was played through the transducers to inform a touch down event. The same haptic feedback was used for all conditions.

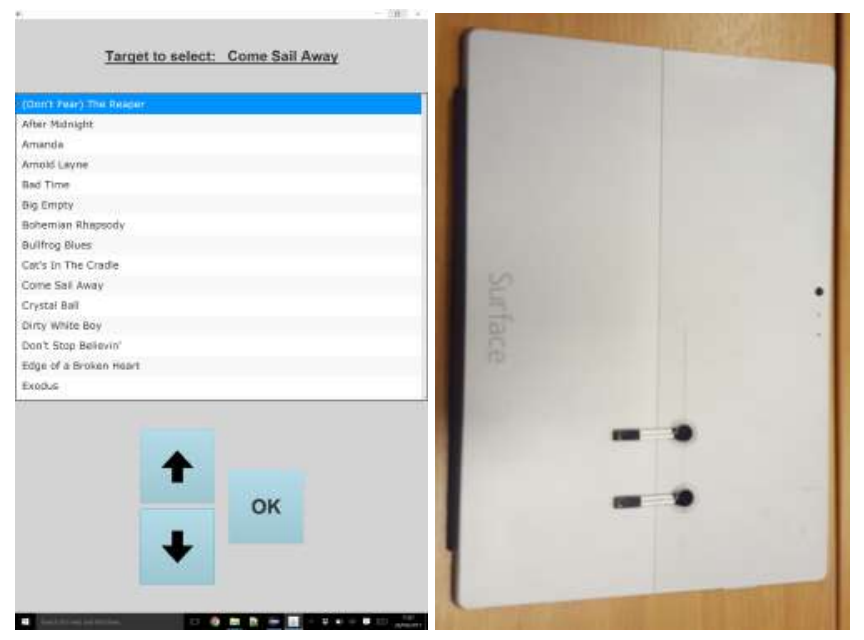

Figure 1. The scrolling task with $8 \mathrm{~mm}$ menu item heights (left). Two Peratech force sensors placed on the back of the touchscreen tablet for the pressure-based buttons (right).

\section{Experimental Design}

We recruited eighteen participants (12 females), aged between 18-32 $($ mean $=22.4, \mathrm{SD}=3.9)$ from the University to take part in the study. Two participants used their left hand for everyday tasks. All participants held a valid driv- 
ing license: UK (13), other European countries (2) and Singapore (2).

The study took no longer than 90 minutes to complete and breaks were given between conditions and when requested to reduce fatigue and travel sickness. The participants were paid $£ 10$ for their time. A training period was given for each condition before the study started to allow the participants to familiarise themselves with the input techniques. A short interview was conducted after the study finished that asked the participants which input techniques were preferred and if haptic feedback subjectively helped their input while inside the moving vehicle.
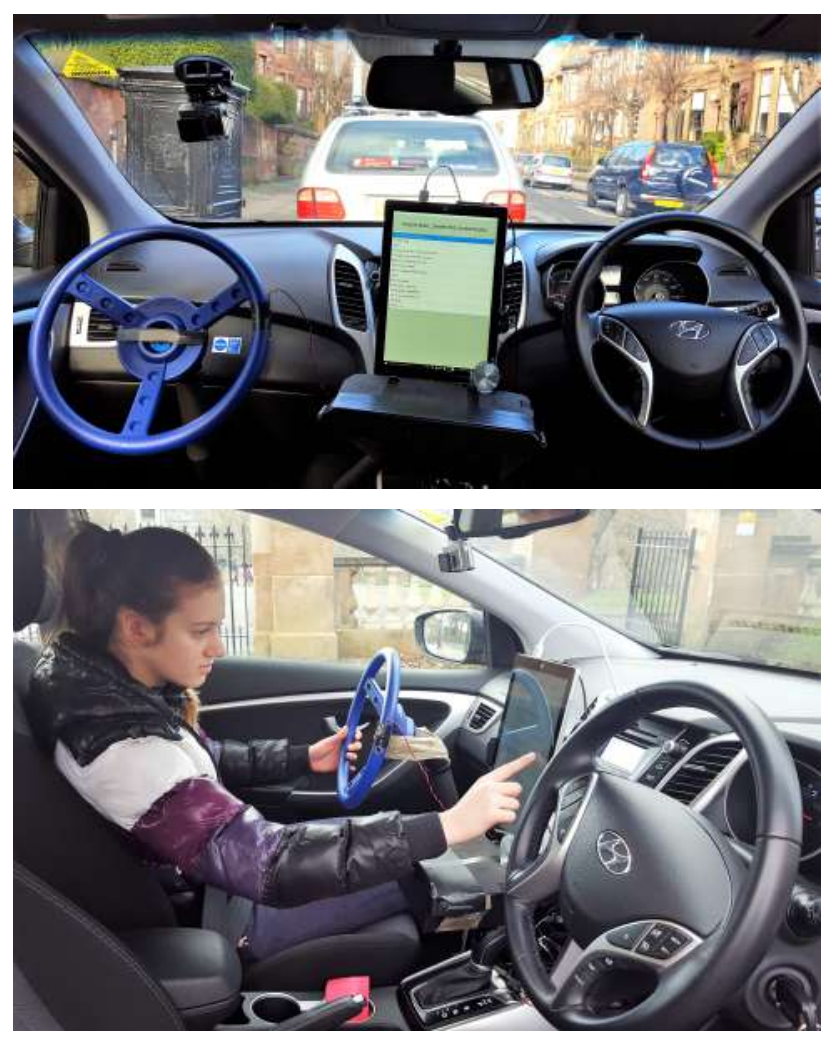

Figure 2. In-car experimental setup (top). A participant performing the scrolling task inside our vehicle (bottom).

A Hyundai 130 with an automatic transmission was hired for the study. An Arkon mount (www.arkon.com) was used to securely hold the hardware in place and the touchscreen tablet was positioned over the car's centre console (see Figure 2). A qualified experimenter drove the vehicle around a pre-defined route in a quiet location in the city to reduce the risk of accidents and avoid areas with large numbers of pedestrians. The route was designed to create a realistic driving scenario so consisted of straight roads, left and right turns and uphill and downhill sections. Traffic light junctions were avoided to eliminate prolonged stationary periods hence decreasing the chances of users performing the task when not experiencing the noise, vibrations and forces of a moving vehicle. The distance of one lap of the route was approximately 0.5 miles and the route was repeated until the end of each condition.

We added a mock steering wheel on the front passenger side to: (1) simulate a real driving position and (2) allow us to measure the time when the right hand lifted off the wheel to start a selection to when it was completed. This gave us a good measure of selection time for each selection.

For the mock steering wheel, a $30 \mathrm{~cm}$ toy wheel was attached to a monopod which was then secured to the car's floor. A Peratech force sensor was attached to the right side of this wheel to detect when the right hand was moved to start the input. For each trial, the participants were asked to hold the wheel with both hands in the standard UK 'ten and two o'clock' driving position when not performing the scrolling task on the touchscreen.

A within-subjects $3 \times 2 \times 2$ (Input Technique $x$ Target Size $x$ Type of Feedback) experimental design was used. There were 12 conditions in total, which were counterbalanced by input technique and then further randomised by a combination of target size and type of feedback to reduce ordering effects. The Independent Variables were Input Technique (Direct Touch, Onscreen Buttons and Pressure Input), Target Size $(4 \mathrm{~mm}$ and $8 \mathrm{~mm}$ ) and Type of Feedback (with and without vibrotactile feedback). The Dependent Variables were the number of correct target selections (\%) and selection time (milliseconds), calculated from when the hand lifted off the steering to when the song displayed was selected. The hypotheses were:

H1: Target accuracy with Onscreen Buttons and Pressure Input will be significantly greater than Direct Touch;

H2: Selection time will be significantly quicker with Direct Touch than the other two input techniques;

H3: Vibrotactile feedback will significantly improve target accuracy and reduce selection time;

H4: The smaller $4 \mathrm{~mm}$ targets will take significantly longer to select and cause more incorrect selections than the $8 \mathrm{~mm}$ targets.

\section{RESULTS}

Fifteen targets were selected for each condition and each participant completed one block per condition. Therefore, a total of 3240 selections (15 targets per block $x 12$ conditions $x 18$ participants) was recorded for the study. The data for target accuracy and selection time did not conform to a normal distribution therefore the Aligned Rank Transform method [30] was applied before performing threefactor (Input Technique, Target Size and Type of Feedback) repeated-measures ANOVAs. The mean target accuracy and selection time for each condition are shown in Figure 3 and Figure 4 respectively.

\section{Target Accuracy}

The three-factor ANOVA for target accuracy showed a significant main effect for Input Technique $F(2,187)=$ 
223.98, $p<0.001$. Post hoc pairwise comparisons showed that Direct Touch was less accurate than both Onscreen Buttons and Pressure Input. There was no difference in accuracy between Onscreen Buttons and Pressure Input. A significant main effect was observed for Target Size $F(1$, $187)=107.69, p<0.001$. Accuracy was lower when selecting the $4 \mathrm{~mm}$ targets than the $8 \mathrm{~mm}$ ones. No significant main effect was found for Type of Feedback $F(1,187)=$ $0.01, p>0.05$.

The interaction between Input Technique and Target Size was significant, $F(2,187)=62.64, p<0.05$. The interaction between Target Size and Type of Feedback was not significant, $F(2,187)=0.11, p>0.05$. The interaction between Input Technique and Type of Feedback was not significant, $F(2,187)=1.01, p>0.05$. The interaction between all three factors was also not significant, $F(2,187)=$ $0.04, p>0.05$. The interactions between the factors are not required to support or reject the hypotheses.

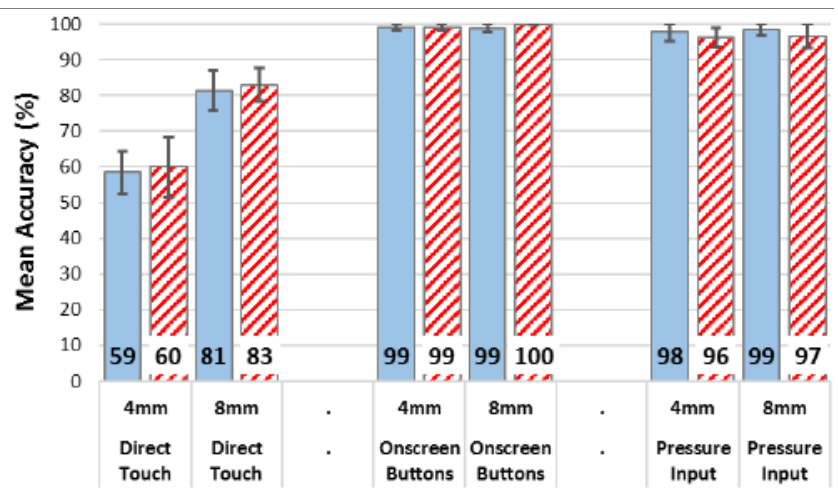

Figure 3. The mean target accuracy (\%) for each condition. The blue solid and red striped bars represent no feedback and with haptic feedback respectively. Error bars denote CI (95\%).

\section{Selection Time}

The results showed a significant main effect for Input Technique $F(2,187)=1294.20, p<0.001$. Post hoc pairwise comparisons showed that Direct Touch took less time than both Onscreen Buttons and Pressure Input. Using the Onscreen Buttons was significantly quicker than Pressure Input. A significant main effect was observed for Target Size $F(1,187)=10.93, p<0.001$. The $4 \mathrm{~mm}$ targets took less time to select than the $8 \mathrm{~mm}$ targets. No significant main effect was found for Type of Feedback $F(1,187)=$ $0.01, p>0.05$.

The interaction between Input Technique and Target Size was not significant, $F(2,187)=0.27, p>0.05$. The interaction between Input Technique and Type of Feedback was not significant, $F(2,187)=0.94, p>0.05$. The interaction between Target Size and Type of Feedback was not significant, $F(2,187)=0.26, p>0.05$. The interaction between all three factors was also not significant, $F(2,187)=0.53, p$ $>0.05$.

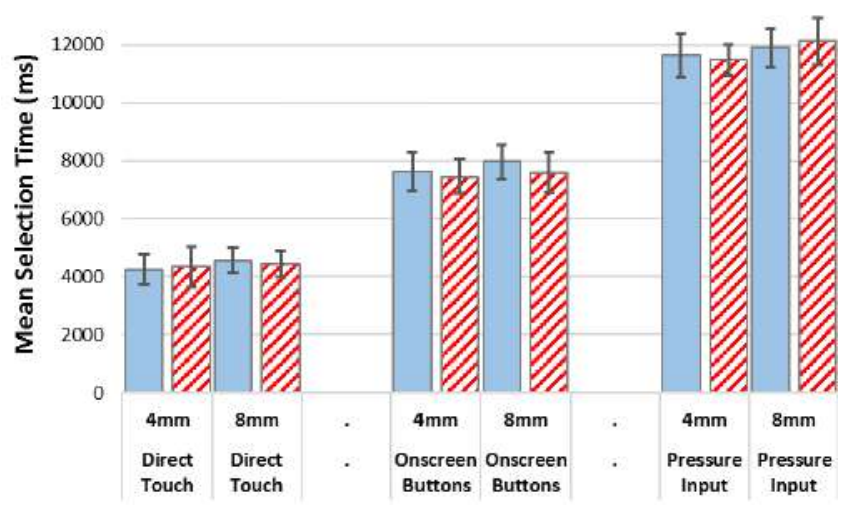

Figure 4. The mean selection time (ms) for each condition.

The blue solid and red striped bars represent no feedback and with haptic feedback respectively. Error bars denote CI $(95 \%)$.

Figure 5 shows a scatter plot of the mean selection time for each item index for Direct Touch while Figure 6 illustrates a plot of the mean selection time for each item for Onscreen Buttons. Figure 7 shows a scatter plot of the mean selection time for each item index for Pressure Input. Note that index 1 is the first item at the top of the list.
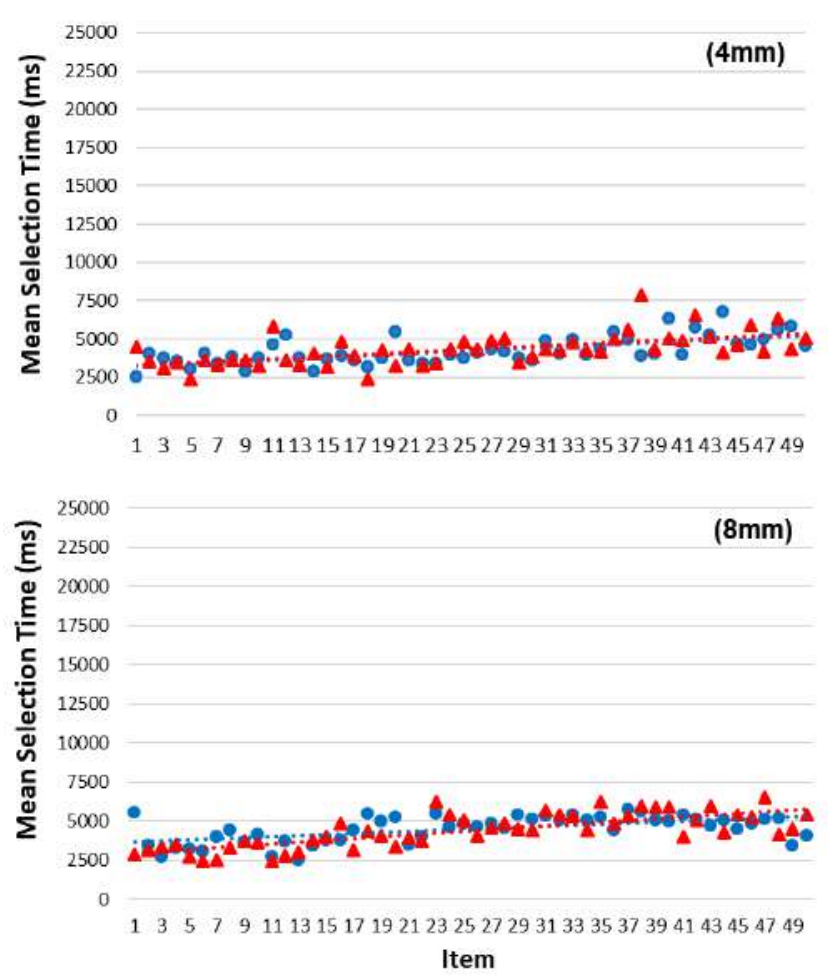

Figure 5. A plot of the mean selection time for each item index in the list for Direct Touch. The blue circles represent no feedback while the red triangles denote the mean selection times with haptic feedback. The top and bottom graphs represent the mean selection times for the $4 \mathrm{~mm}$ and $8 \mathrm{~mm}$ menu item heights respectively. Note: index 1 is the first item at the top of the list. This is the same for Figure 6 and Figure 7. 

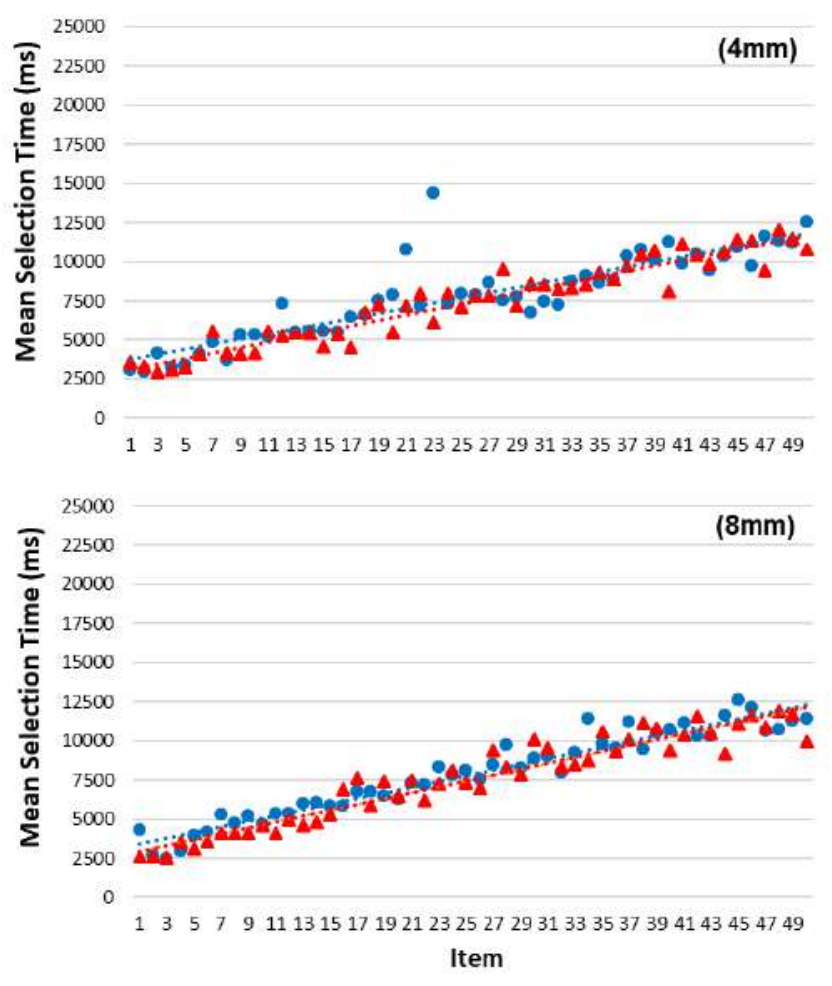

Figure 6. A plot of the mean selection time for each item index for Onscreen Buttons. The blue circles represent no feedback while the red triangles denote the mean selection times with haptic feedback.

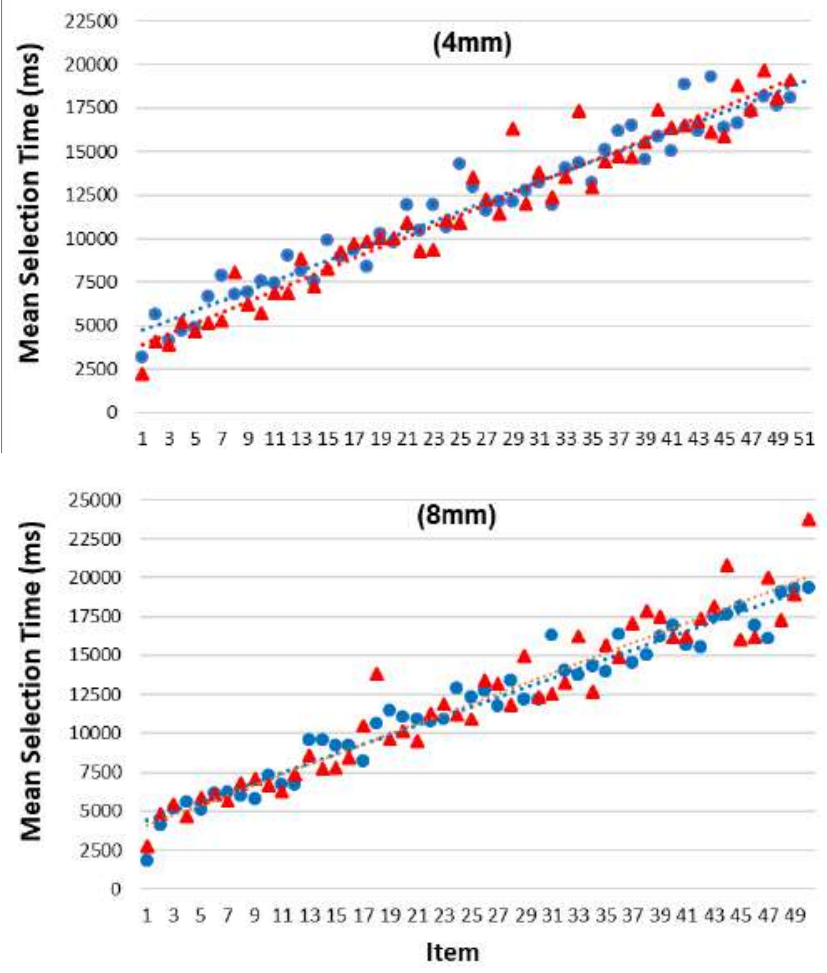

Figure 7. A plot of the mean selection time for each item index for Pressure Input.
The table in Figure 8 shows the coefficient of determination $\left(R^{2}\right)$ and correlation coefficient $(R)$ for each condition in the plots in Figure 5, Figure 6 and Figure 7. It is also noting that all indexes were selected at least twice across all conditions but the number of selections for each item in the list was not equal since the trials were pseudo-randomly chosen. As a result, selection time might be skewed for those items that were selected the least number of times.

\begin{tabular}{|l|l|l|c|c|}
\hline $\begin{array}{c}\text { Input } \\
\text { Technique }\end{array}$ & Target Size & \multicolumn{1}{|c|}{$\begin{array}{c}\text { Type of } \\
\text { Feedback }\end{array}$} & $\mathbf{R}^{2}$ & R \\
\hline $\begin{array}{l}\text { Direct } \\
\text { Touch }\end{array}$ & $4 \mathrm{~mm}$ & No feedback & 0.427 & 0.653 \\
\hline $\begin{array}{l}\text { Direct } \\
\text { Touch }\end{array}$ & $4 \mathrm{~mm}$ & Haptic Feedback & 0.354 & 0.595 \\
\hline $\begin{array}{l}\text { Direct } \\
\text { Touch }\end{array}$ & $8 \mathrm{~mm}$ & No feedback & 0.301 & 0.549 \\
\hline $\begin{array}{l}\text { Direct } \\
\text { Touch }\end{array}$ & $8 \mathrm{~mm}$ & Haptic Feedback & 0.567 & 0.753 \\
\hline \hline $\begin{array}{l}\text { Onscreen } \\
\text { Buttons }\end{array}$ & $4 \mathrm{~mm}$ & No feedback & 0.754 & 0.868 \\
\hline $\begin{array}{l}\text { Onscreen } \\
\text { Buttons }\end{array}$ & $4 \mathrm{~mm}$ & Haptic Feedback & 0.918 & 0.958 \\
\hline $\begin{array}{l}\text { Onscreen } \\
\text { Buttons }\end{array}$ & $8 \mathrm{~mm}$ & No feedback & 0.933 & 0.966 \\
\hline $\begin{array}{l}\text { Onscreen } \\
\text { Buttons }\end{array}$ & $8 \mathrm{~mm}$ & Haptic Feedback & 0.919 & 0.959 \\
\hline $\begin{array}{l}\text { Pressure } \\
\text { Input }\end{array}$ & $4 \mathrm{~mm}$ & No feedback & 0.944 & 0.972 \\
\hline $\begin{array}{l}\text { Pressure } \\
\text { Input }\end{array}$ & $4 \mathrm{~mm}$ & Haptic Feedback & 0.944 & 0.972 \\
\hline $\begin{array}{l}\text { Pressure } \\
\text { Input }\end{array}$ & $8 \mathrm{~mm}$ & No feedback & 0.953 & 0.976 \\
\hline $\begin{array}{l}\text { Pressure } \\
\text { Input }\end{array}$ & $8 \mathrm{~mm}$ & 0.922 & 0.960 \\
\hline
\end{tabular}

Figure 8. A table showing the coefficient of determination $\left(R^{2}\right)$ and correlation coefficient $(R)$ for each condition in the plots in Figure 5, Figure 6 and Figure 7.

In general, a weak to medium correlation was found between selection time and item index for Direct Touch $\left(R^{2}\right.$ was between $0.549-0.753$ ), thus it is more difficult to predict the selection time based on the position of the item in the list. For Onscreen Buttons, a medium to strong correlation was found between selection time and item index $\left(R^{2}\right.$ was between $0.868-0.966$ ). The items at the end of the list took approximately $12500 \mathrm{~ms}$ when using the onscreen buttons, compared to between $2000-4000 \mathrm{~ms}$ for the items at the start of the list. A strong correlation between selection time and item index was found for Pressure Input across both target size and with or without haptic feedback $\left(R^{2}>\right.$ $0.959)$. The items at the end of the list took approximately $20000 \mathrm{~ms}$ to select when using the pressure-based buttons, compared to around $3000-5000 \mathrm{~ms}$ for the menu items at the top of the list. 


\section{DISCUSSIONS}

The results for target accuracy showed that Direct Touch was less accurate than both Onscreen Buttons and Pressure Input, therefore hypothesis $\mathrm{H} 1$ is supported. Our previous in-car experiment [21] found that accuracy was high $(>94 \%)$ between touch input, pressure input and when using a physical dial for selecting $16 \mathrm{~mm}$ menu item heights. It was anticipated that users in the study presented in this paper would make more incorrect selections since smaller $8 \mathrm{~mm}$ and $4 \mathrm{~mm}$ menu heights were tested, especially with Direct Touch since errors cannot be corrected while users could correct overshoots with Onscreen Buttons and Pressure Input. Further analysis was done on the incorrect target selections for the Direct Touch conditions and for the $4 \mathrm{~mm}$ heights, $92 \%$ of them were missed by one menu position while $94 \%$ of the $8 \mathrm{~mm}$ menu heights were incorrectly selected by one position regardless of feedback type.

Accuracy was low for Direct Touch, particularly when selecting the $4 \mathrm{~mm}$ targets $(59 \%$ for input without haptic feedback), perhaps due to the finger occluding the target area which led to ambiguous selection (described as the 'fat finger' problem [3]). For comparison, our previous work [21] reported an accuracy of $95 \%$ for Direct Touch without haptic feedback where users were also driving on public roads but menu items were taller at $16 \mathrm{~mm}$. Combining the results on target accuracy from our previous work and this study suggests that the number of correct selections declines quickly as target size falls - 16mm: $95 \%, 8 \mathrm{~mm}$ : $81 \%$ and $4 \mathrm{~mm} \mathrm{59 \%}$. Care should be taken when choosing target sizes for touchscreens to ensure accurate input in driving situations.

The results for selection time between the input techniques showed that Direct Touch was quicker than Onscreen Buttons and Pressure Input, therefore hypothesis H2 is support. Directly selecting the targets in the list was almost twice as fast as using the onscreen arrow buttons and nearly three times quicker than the pressure-based buttons. There is an apparent speed vs. accuracy trade-off for Direct Touch but an incorrect is likely to take more time to recover from the error: accuracy is poor but selection time is fast. The issue then becomes the cost of recovering from the erroneous selection. If the cost of an incorrect selection is low then this technique can be effective, but if the recovery cost is high then this technique might be less suitable for in-car use.

Input using the onscreen buttons took less time than using pressure input, similar to the findings from our earlier work $[20,21]$. For comparison, the overall selection time for Onscreen Buttons without haptic feedback was very similar to our previous results [21] despite participants in the study presented in this paper having to scroll through a much longer list. However, users in our previous experiment were also driving, which is a limitation of our current study. We predict that selection time will increase further when using onscreen buttons for a long menu while driving.
Pressure-based buttons was slower than the results reported in our previous work $[20,21]$ which was already the slowest input technique when compared to direct touch selection and a physical dial. Selection time with our design of pressure-based buttons is likely to take even longer if users are also driving, so it is difficult to recommend the current implementation of pressure input for quick targeting with list interfaces on in-car touchscreens.

Target accuracy when selecting the $4 \mathrm{~mm}$ menu items was lower than the $8 \mathrm{~mm}$ heights. The largest difference in terms of accuracy between the target sizes was for Direct Touch as accuracy was greater than $96 \%$ for both Onscreen Buttons and Pressure Input regardless of feedback type. We anticipated that accuracy would drop when selecting the targets directly in the list as target height is reduced but wanted to test the limits and see how much performance decreased while on the move.

The results also showed that the $4 \mathrm{~mm}$ menu items took less time to select than the $8 \mathrm{~mm}$ ones and therefore hypothesis H4 cannot be fully supported and is rejected. It is worth noting that the difference in selection time was marginal between the two target sizes. We predicted that the $4 \mathrm{~mm}$ targets would take more time to select than the $8 \mathrm{~mm}$ targets but perhaps the smaller menu items were quicker to search due to more items were shown onscreen and therefore less scrolling was required. Maybe it is beneficial to reduce target size to display more items onscreen for in-car list interfaces without a heavy cost to input speed.

No statistical significant differences were found for both target accuracy and selection time between with and without haptic feedback. Therefore, hypothesis H3 is rejected. We asked the participants whether they preferred having vibrotactile feedback for input and 13 out of 18 participants commented that it was useful in terms of knowing that the touchscreen had acknowledge some form of input. The remaining participants felt that haptic feedback made no difference to their selections on the touchscreen. One limitation with our hardware setup was that only one surface transducer was used. As a result, vibrotactile feedback on the touchscreen might have been more difficult for some users to perceive, especially inside a moving vehicle where ambient noise and vibrations are likely to counteract the haptic feedback.

The graphs in Figure 5, Figure 6 and Figure 7 gives some broad indication on the input time required for each technique based on the item position in the list. A weak correlation between selection time and item position was found for direct input, so it is more difficult to predict the speed of input for longer lists. Selection time with direct input for the items at the end of the list was much quicker than both the onscreen buttons and pressure input. Selection time using onscreen buttons for long menus might improve with side scroll bars to allow for faster page access. We removed scroll bars from our list interface to avoid adding 
noise to selection time and allow a fairer comparison between the input techniques.

Pressure-based buttons took the longest to select the items at the end of the list and needs further refinement for it to be effective in driving situations. We decreased the speed of the cursor, when compared to our previous studies [20, $21]$, to avoid constantly overshooting the current target to select in the list. Perhaps a dynamic mapping between the input pressure range and the speed of the cursor would significantly improve selection time for menu items near the end of lists. We plan to investigate this in the future.

The participants were asked which input technique they preferred to use and seven participants suggested direct scrolling while eight participants liked onscreen buttons and the remaining three users chose pressure input. Despite poor target accuracy, participants liked direct scrolling because they felt "it was quicker" than the other two input techniques and that they were familiar with the technique from using their mobile phones and touchscreen devices every day. In the experiment, there was no cost to missing a target. If there were, participants may have had a different view of their preferred technique. This will be studied in future experiments.

The participants who preferred onscreen buttons suggested it was easier, especially with haptic feedback, to count the number of button presses to select an item once they had scrolled and found the target on the screen. The participants therefore suggested that onscreen buttons would be less visually demanding to use in realistic driving situations. Furthermore, it gives users a chance to correct their input if the cursor overshot the target to select in the list. Onscreen pressure-based buttons were subjectively the least preferred input technique. The participants said that they did not like applying force on the touchscreen for long periods and felt it was the least safe method to use while driving. In addition, pressure input was also the slowest input technique so our design cannot be recommended for in-car scrolling tasks and applications. Perhaps pressure input is more effective for simpler touchscreen actions such as differentiating between standard and harder taps, similar to the interactions found on Apple iPhones. Future work should explore different forms of pressure-based interactions as force-sensing touchscreens and touch-surfaces become more prevalent on dashboards and centre consoles.

\section{CONCLUSIONS}

Car manufacturers are developing new ways for drivers to interact with centre console controls and infotainment systems. One common method is with the use of touchscreens and they are becoming a standard method for drivers to change the climate control, select a song from a playlist and choose a location on a map, for example. We conducted an on-the-road study which evaluated and compared the performance of three different types of input techniques for scrolling tasks on touchscreens. Our study is also the first to evaluate pressure input for scrolling interfaces on touchscreens in non-driving, in-car situations.

The results showed that direct scrolling was the quickest method but resulted in poor target accuracy, especially when selecting targets that were $4 \mathrm{~mm}$ in height. Input with onscreen buttons caused fewer incorrect selections than direct scrolling as accuracy was near $100 \%$ regardless of target size, but selection time took almost twice as long. Pressure-based onscreen buttons also resulted in very high accuracy but selection times took even longer and this technique was subjectively the least preferred. Haptic feedback had no effect on scrolling performance but was subjectively preferred by a majority of the users, suggesting vibrotactile cues have value for touchscreen interactions in driving contexts. List-based scrolling tasks are very common with touchscreen interfaces and we hope the findings in this paper will inform designers to create more useful and less visually demanding in-car systems and applications.

\section{ACKNOWLEDGEMENTS}

This work was funded by the H2020 HAPPINESS project (Grant agreement no. 645145).

\section{REFERENCES}

[1] National H.T.S. 2012. Visual-Manual NHTSA Driver Distraction Guidelines for In-Vehicle Electronic Devices.

[2] Ahmad, B.I., Langdon, P.M., Godsill, S.J., Hardy, R. Skrypchuk, L. and Donkor, R. 2015. Touchscreen usability and input performance in vehicles under different road conditions: an evaluative study. In Proceedings of the 7th International Conference on Automotive User Interfaces and Interactive Vehicular Applications - AutomotiveUI '15, 47-54.

[3] Baudisch, P. and Chu, G. 2009. Back-of-device interaction allows creating very small touch devices. In Proceedings of the SIGCHI conference on Human factors in computing systems - CHI '09, 1923-1932.

[4] BMW 7 Series Innovations: http:/www.bmw.com/com/en/newvehicles/7series/se dan/2015/showroom/innovative_functionality.html.

[5] Brewster, S. and Hughes, M. 2009. Pressure-based text entry for mobile devices. In Proceedings of the 11th International Conference on Human-Computer Interaction with Mobile Devices and Services MobileHCI '09, 1-4.

[6] Burnett, G., Crundall, E., Large, D., Lawson, G. and Skrypchuk, L. 2013. A study of unidirectional swipe gestures on in-vehicle touch screens. Proceedings of the 5th International Conference on Automotive User Interfaces and Interactive Vehicular Applications AutomotiveUI'13, 22-29.

[7] Diwischek, L. and Lisseman, J. 2015. Tactile Feedback for Virtual Automotive Steering Wheel Switches. In Proceedings of the 7th International 
Conference on Automotive User Interfaces and Interactive Vehicular Applications - AutomotiveUI '15, 31-38.

[8] Ecker, R., Broy, V., Hertzschuch, K. and Butz, A. 2010. Visual cues supporting direct touch gesture interaction with in-vehicle information systems. In Proceedings of the 2nd International Conference on Automotive User Interfaces and Interactive Vehicular Applications - AutomotiveUI '10, 80-87.

[9] Goel, M., Wobbrock, J. and Patel, S. 2012. GripSense: using built-in sensors to detect hand posture and pressure on commodity mobile phones. In Proceedings of the 25th annual ACM symposium on User interface software and technology - UIST '12, 545-554.

[10] Heo, S. and Lee, G. 2012. ForceDrag: using pressure as a touch input modifier. In Proceedings of the 24th Australian Computer-Human Interaction Conference - OZCHI 2012, 2012, 204-207.

[11] Hoggan, E., Stewart, C., Haverinen, L., Jacucci, G. and Lantz, V. 2012. Pressages: Augmenting Phone Calls with Non-Verbal Messages. In Proceedings of the 25th annual ACM symposium on User interface software and technology - UIST '12 (2012), 555-562.

[12] Holmen, J.K. and Zadeh, M.H. 2010. Effects of varying haptic feedback on driver distraction during vehicular window adjustment. In Proceedings of the 2nd International Conference on Automotive User Interfaces and Interactive Vehicular Applications AutomotiveUI '10, 88-91.

[13] Huber, J., Sheik-Nainar, M. and Matic, N. 2016. Towards an Interaction Language for Force-enabled Touchpads in Cars. In Adjunct Proceedings of the 8th International Conference on Automotive User Interfaces and Interactive Vehicular Applications AutomotiveUI '16, 195-200.

[14] Kern, D., Mahr, A., Castronovo, S., Schmidt, A. and Müller, C. 2010. Making use of drivers' glances onto the screen for explicit gaze-based interaction. In Proceedings of the 2nd International Conference on Automotive User Interfaces and Interactive Vehicular Applications - AutomotiveUI '10, 110-113.

[15] Kujala, T. 2013. Browsing the information highway while driving: Three in-vehicle touch screen scrolling methods and driver distraction. Personal and Ubiquitous Computing. 17, 5 (2013), 815-823.

[16] Lasch, A. and Kujala, T. 2012. Designing browsing for in-car music player: effects of touch screen scrolling techniques, items per page and screen orientation on driver distraction. In Proceedings of the 4th International Conference on Automotive User Interfaces and Interactive Vehicular Applications AutomotiveUI '12, 41-48.
[17] Lee, J.D., Roberts, S.C., Hoffman, J.D. and Angell, L.S. 2012. Scrolling and Driving. Human Factors. 54, 2 (2012), 250-263.

[18] McLachlan, R., Boland, D. and Brewster, S. 2014. Transient and transitional states: pressure as an auxiliary input modality for bimanual interaction. In Proceedings of the 32nd annual ACM conference on Human factors in computing systems - CHI '14 (2014), 401-410.

[19] McLachlan, R. and Brewster, S. 2015. Bimanual input for tablet devices with pressure and multi-touch gestures. In Proceedings of the 17th International Conference on Human-Computer Interaction with Mobile Devices and Services - MobileHCI '15, 547556.

[20] Ng, A. and Brewster, S.A. 2016. Investigating Pressure Input and Haptic Feedback for In-Car Touchscreens and Touch Surfaces. In Proceedings of the 8th International Conference on Automotive User Interfaces and Interactive Vehicular Applications, AutomotiveUI' 16, 121-128.

[21] Ng, A., Brewster, S.A., Beruscha, F. and Krautter, W. 2017. An Evaluation of Input Controls for In-Car Interactions. In Proceedings of the 2017 CHI Conference on Human Factors in Computing Systems - $C H I^{\prime} 17,2845-2852$.

[22] Pitts, M.J., Burnett, G., Skrypchuk, L., Wellings, T., Attridge, A. and Williams, M. a. 2012. Visual-haptic feedback interaction in automotive touchscreens. Displays. 33, 1 (2012), 7-16.

[23] Pitts, M.J., Williams, M.A. and Attridge, A. 2009. Assessing Subjective Response to Haptic Feedback in Automotive Touchscreens. In Proceedings of the 1st International Conference on Automotive User Interfaces and Interactive Vehicular Applications AutomotiveUI '09, 11-18.

[24] Ramos, G., Boulos, M. and Balakrishnan, R. 2004. Pressure Widgets. In Proceedings of the 2004 conference on Human factors in computing systems CHI '04, 487-494.

[25] Richter, H., Ecker, R., Deisler, C. and Butz, A. 2010. HapTouch and the $2+1$ state model: potentials of haptic feedback on touch based in-vehicle information systems. In Proceedings of the 2nd International Conference on Automotive User Interfaces and Interactive Vehicular Applications - AutomotiveUI '09, 72-79.

[26] Rümelin, S. and Butz, A. 2013. How to make large touch screens usable while driving. In Proceedings of the 5th International Conference on Automotive User Interfaces and Interactive Vehicular Applications AutomotiveUI'13, 48-55.

[27] Rydström, A., Grane, C. and Bengtsson, P. 2009. 
Driver behaviour during haptic and visual secondary tasks. In Proceedings of the lst International Conference on Automotive User Interfaces and Interactive Vehicular Applications, 121-127.

[28] Spelmezan, D., Appert, C., Chapuis, O. and Pietriga, E. 2013. Side pressure for bidirectional navigation on small devices. In Proceedings of the 15th International conference on Human-computer interaction with mobile devices and services MobileHCI '13, 11-20.

[29] Stewart, C., Rohs, M., Kratz, S. and Essl, G. 2010. Characteristics of pressure-based input for mobile devices. In Proceedings of the 28th international conference on Human factors in computing systems CHI '10, 801-810.

[30] Wobbrock, J.O., Findlater, L., Gergle, D. and Higgins, J.J. 2011. The aligned rank transform for nonparametric factorial analyses using only anova procedures. In Proceedings of the SIGCHI Conference on Human Factors in Computing Systems - CHI '11, 143-146. 